\title{
A translation invariant pure DEA model
}

\author{
Vincent Charles $^{a^{*}}$, Rolf Färe ${ }^{b}$, and Shawna Grosskopf ${ }^{\mathfrak{c}}$ \\ ${ }^{a}$ CENTRUM Católica Graduate Business School, PUCP, Jr. Daniel Alomía Robles 125-129, Los Álamos \\ de Monterrico, Santiago de Surco, Lima 33, Peru \\ vcharles@pucp.pe \\ ${ }^{\mathrm{b}}$ Department of Economics, Oregon State University, Corvallis, OR 97331, United States \\ rolf.fare@oregonstate.edu \\ ${ }^{\mathrm{c}}$ Department of Economics, Oregon State University, Corvallis, OR 97331, United States \\ Shawna.grosskopf@oregonstate.edu
}

\begin{abstract}
This communication complements the DEA model proposed by Lovell and Pastor (Eur. J. Oper. Res. 118 (1999), 46-51), by incorporating both positive and negative criteria in the model. As such, we propose a DEA model, known as pure DEA, using a directional distance function approach.
\end{abstract}

Keywords: data envelopment analysis; invariance; directional distance function.

\section{Introduction}

Decisions are an integral part of human life. Regardless of the variety of problems that need to be solved, it usually happens that one must evaluate several alternatives and then choose among them. Data Envelopment Analysis (DEA) facilitates such an analysis, and, furthermore, it allows the ordering of the alternatives (i.e., decision-making units, DMUs).

By definition, DEA models have inputs and outputs; pure DEA refers to a class of models wherein either inputs only or outputs only are considered (Lovell and Pastor, 1997). Lovell and 
Pastor (1999) showed that a constant returns-to-scale model without inputs (or without outputs) is meaningless; thus, the intensity variables need to be constrained, i.e., $\sum_{k=1}^{K} z_{k}=1$. Hence, they considered radial DEA models with a single constant input (output) and radial DEA models without inputs (or without outputs) so as to accommodate situations that arise in some multicriteria decision-making problems. These models admit only positive (or negative) criteria. This means that either all of the criteria under evaluation are positive or negative.

However, in some situations one may encounter both positive and/or negative evaluations [variables], which warrant the translation invariance property to be satisfied. Furthermore, these situations turn out to be quite frequent in real life. For instance, a personnel selection problem needs the evaluation of some of the following positive $(+)$ and negative $(-)$ criteria: years of experience $(+)$, level of qualification $(+)$, and salary (-). Similarly, a vacation destination problem might be assessed based on the criteria: entertainment options $(+)$, facilities $(+)$, travel cost $(-)$, and accommodation cost (-). An automobile selection problem is yet another situation in which criteria such as price $(-)$, mileage $(+)$, and quality $(+)$ could be considered for evaluation. The same kind of situations may also arise in the development of indexes of social and economic development, such as the doing business index and the regional competitiveness index, among others.

In this context, the present paper complements the work done by Lovell and Pastor (1999), by incorporating both positive and negative criteria in the model. 


\section{Modeling}

The input-oriented model in line with Lovell and Pastor (1999) can be written as follows:

$\operatorname{Min} \lambda$

subject to

$\sum_{k=1}^{K} z_{k} x_{k n} \leqq \lambda x_{k_{o}}, n=1,2, \ldots, N$

$z_{k} \geqq 0, k=1,2, \ldots, K, \sum_{k=1}^{K} z_{k}=1$.

However, as previously mentioned, there are situations that require both positive and/or negative evaluations, which warrant the translation invariance property to be satisfied.

System (1) is unit invariant, but not translation invariant. Färe and Grosskopf (2013) showed that the following model is translation invariant:

$\operatorname{Max} \beta$

subject to

$\sum_{k=1}^{K} z_{k} x_{k n} \leqq x_{k_{o} n}-\beta g_{n}, n=1,2, \ldots, N$

$\sum_{k=1}^{K} z_{k} y_{k m} \geqq y_{k_{o} m}, m=1,2, \ldots, M$,

$z_{k} \geqq 0, k=1,2, \ldots, K, \sum_{k=1}^{K} z_{k}=1$,

where $g=\left(g_{1,} g_{2}, \ldots, g_{N}\right) \geq 0, g \neq 0$ is the directional input vector which specifies the direction in which data are projected to the frontier of technology or, more precisely, as Aparicio et al. (2016) showed that the g vector must be translation invariant. When we remove the output constraints from System (2) we have a pure input-oriented model: 
$\operatorname{Max} \beta$

subject to

$\sum_{k=1}^{K} z_{k} x_{k n} \leqq x_{k_{o} n}-\beta g_{n}, n=1,2, \ldots, N$,

$z_{k} \geqq 0, k=1,2, \ldots, K, \sum_{k=1}^{K} z_{k}=1$,

System (3) is both unit and translation invariant (See Appendix); given that $g_{n}$ is exogenous to the data, then it is also invariant with respect to an affine data transformation. In order to incorporate both positive and negative evaluations in System (3), we shall split the $N$ input constraints into two sets, say $N=N_{1}+N_{2}$.

Let $\left[x^{+}\right]_{\mathrm{KxN}_{1}}$ be an evaluation matrix consisting of $M$ alternatives (DMUs) and $N_{1}$ positive criteria (values) and let $\left[x^{-}\right]_{\mathrm{KxN}_{2}}$ be an evaluation matrix consisting of $M$ alternatives (DMUs) and $N_{2}$ negative criteria. We have two options: to either transform the negative criteria (values) into positive criteria (values) or vice versa. Let us transform the positive criteria evaluation matrix $\left[x^{+}\right]_{\mathrm{KxN}_{1}}$ into negative criteria by allowing an affine transformation. Then, the $n_{l}^{\text {th }}$ criteria (values) of the matrix can be transformed as $0 \leqq x_{k n_{1}}^{-^{\prime}}=a_{n_{1}} x_{k n_{1}}^{+^{\prime}}+b_{n_{1}}$, where $a_{n_{1}}$ is negative. Taking account of both the positive and negative evaluations, System (4) can be obtained from System (3) based on the above discussion:

$\operatorname{Max} \beta-\gamma$

subject to

$\sum_{k=1}^{K} z_{k} x_{k n_{1}}^{-^{\prime}} \geqq x_{k_{o} n_{1}}^{-^{\prime}}-\gamma g_{n_{1}}^{\prime}, n_{1}=1,2, \ldots, N_{1}$,

$\sum_{k=1}^{K} z_{k} x_{k n_{2}}^{-} \leqq x_{k_{o} n_{2}}^{-}-\beta g_{n_{2}}, n_{2}=1,2, \ldots, N_{2}$,

$z_{k} \geqq 0, k=1,2, \ldots, K, \sum_{k=1}^{K} z_{k}=1$, 
where $0 \leq g_{n_{1}}^{\prime}=a_{n_{1}} g_{n_{1}}$ and $g=\left(g_{1}^{\prime}, g_{2}^{\prime}, \ldots, g_{N_{1}}^{\prime}, g_{1}, g_{2}, \ldots, g_{N 2}\right) \geq 0, g \neq 0$ is the directional input vector which specifies the direction in which data are projected to the frontier of technology, and the efficiency indicator for System (4) is $\beta+\gamma$ and it is to be noted that both $\beta$ and $\gamma$ are unit free.

To relate our model in System (4) to the model of Lovell and Pastor in System (1): in System (4) by deleting the first inequality constraint and $\gamma$ in the objective function; then, by taking $g_{n_{2}}, n_{2}=1,2, \ldots, N_{2}$, equal to $x_{k_{o} n_{2}}^{-}$to obtain $x_{k_{o} n_{2}}^{-}(1-\beta)$ on the right-hand-side; finally, by setting $(1-\beta)=\lambda$, and adjusting the objective function so as to obtain $1+\operatorname{Min} \lambda$, the relation to Lovell and Pastor's model is established.

\section{Numerical Example}

The following example demonstrates the applicability of the above sentences of System (4) numerically:

Let us assume that a potential costumer is looking forward to purchasing an automobile. His choice must be made among the given six alternatives (DMUs), represented below by A, B, C, D, E, and F. He needs to compare their performance characteristics in order to find the best options. The criteria considered could be: the price of the automobile (-), the mileage $(+)$, and the quality $(+)$. The data is as defined in the below Table 1, where the numbers provided have been chosen for reasons of simplicity of understanding. As such, we show how we can compare these automobiles and choose the best among the given alternatives. 
Table 1: One negative criterion (Price) and two positive criteria (Mileage and Quality)

\begin{tabular}{|c|c|c|c|c|c|}
\hline $\begin{array}{c}\text { Alternatives } \\
\text { (DMU) } \\
k\end{array}$ & $\begin{array}{c}\text { Price } \\
x_{k 1}^{-}\end{array}$ & $\begin{array}{c}\text { Original } \\
\text { Mileage } \\
x_{k 2}^{+}\end{array}$ & $\begin{array}{c}\text { Transformed } \\
\text { Mileage } \\
x_{k 2}^{-^{\prime}}=-1 . x_{k 2}^{+}+7\end{array}$ & $\begin{array}{c}\text { Original } \\
\text { Quality } \\
x_{k 3}^{+}\end{array}$ & $\begin{array}{c}\text { Transformed } \\
\text { Quality } \\
x_{k 3}^{-^{\prime}}=-1 . x_{k 3}^{+}+10\end{array}$ \\
\hline A & 10 & 0 & 7 & 8 & 2 \\
\hline B & 5 & 6 & 1 & 6 & 4 \\
\hline $\mathrm{C}$ & 8 & 5 & 2 & 7 & 3 \\
\hline D & 3 & 6 & 1 & 9 & 1 \\
\hline $\mathbf{E}$ & 2 & 6 & 1 & 8 & 2 \\
\hline $\mathrm{F}$ & 4 & 4 & 3 & 9 & 1 \\
\hline
\end{tabular}

\begin{tabular}{ccccc}
\hline $\begin{array}{c}\text { Alternatives } \\
\text { (DMU) }\end{array}$ & $\beta$ & $\gamma$ & $\begin{array}{c}\text { Objective } \\
\text { Function } \\
\beta\end{array}$ & $\begin{array}{c}\text { Efficiency } \\
\text { Indicator } \\
\beta-\gamma\end{array}$ \\
\hline A & 8 & 6 & 2 & $14(6)$ \\
B & 3 & 2 & 1 & $5(4)$ \\
C & 6 & 1 & 5 & $7(5)$ \\
D & 1 & 0 & 1 & $1(2)$ \\
E & $\mathbf{0}$ & $\mathbf{0}$ & $\mathbf{0}$ & $\mathbf{0}(1)$ \\
F & 2 & 2 & 0 & $4(3)$ \\
\hline
\end{tabular}

It is to be noted that in the case of multiple optimal solutions, one should select the minimum (maximum) of all the sets $\beta+\gamma$ among all the optimal solutions, under the optimistic (pessimistic) approach towards the DMU of interest. One may also think of using the weighted average of the values obtained in the optimistic and pessimistic approaches. For instance, let us assume that DMU-A has alternative optimal solutions $(8,6)$ and $(16,14)$. In such a case, the optimistic, pessimistic, and weighted average (assuming equal weights) approaches yield an efficiency indicator of 14,30 , and 22 , respectively. In consequence, depending on the approach, the order of the DMUs varies, which is inevitable in multi-criteria decision-making. 


\section{References}

Aparicio, J., Pastor, J. T., \& Vidal, F. (2016). The Directional Distance Function and the Translation Invariance Property, Omega - The International Journal of Management Science, 58, 1-3.

Färe, R., \& Grosskopf, S. (2013). DEA, directional distance functions and positive, affine data transformation. Omega - The International Journal of Management Science, 41(1), 28-30.

Lovell, C. A. K., \& Pastor, J. T. (1997). Target setting: an application to a bank branch network. European Journal of Operations Research, 98(2), 290-299.

Lovell, C. A. K., \& Pastor, J. T. (1999). Radial DEA models without inputs or without outputs. European Journal of Operations Research, 118(1), 46-51.

\section{Appendix}

To verify that (2) is both unit and translation invariant let us look at the $\mathrm{n}^{\text {th }}$ input

$\sum_{k=1}^{K} z_{k} x_{k n} \leqq x_{k^{\prime} n}-\beta g_{n}$

Note that $g_{n}$ is exogenous to the data. Change the unit by $a$ and translate the data by $b$, then

$\sum_{k=1}^{K} z_{k}\left(a x_{k n}+b\right) \leqq\left(a x_{k^{\prime} n}+b\right)-a \beta g_{n}$

remembering that $g_{n}$ has the same unit of measurement as $x_{k^{\prime} n}$. Now,

$\sum_{k=1}^{K} z_{k} a x_{k n}+\sum_{k=1}^{K} z_{k} b \leqq a x_{k^{\prime} n}+b-\beta a g_{n}$,

and by variable returns-to-scale, $\sum_{k=1}^{K} z_{k}=1$,

$$
a \sum_{k=1}^{K} z_{k} x_{k n} \leqq a\left(x_{k^{\prime} n}-\beta g_{n}\right)
$$

and since $a$ cancels we have our original expression (1'). 


\title{
Annex Information: Acceptance Information
}

\author{
Forwarded message \\ De: Robert Dyson <Robert.Dyson@wbs.ac.uk> \\ Date: lun., 21 sept. 2015 a las 11:39 \\ Subject: Your Submission EJOR-D-15-00523R2 \\ To: <vcharles@pucp.pe>,<v.chals@gmail.com> \\ Cc: <rolf.fare@oregonstate.edu>,<Shawna.grosskopf@oregonstate.edu>
}

Ref.: Ms. No. EJOR-D-15-00523R2

A translation invariant pure DEA model

European Journal of Operational Research

Dear Prof. Vincent Charles,

I am pleased to inform you that your paper has been accepted for publication in the European Journal of Operational Research.

The PDF file of the final version of your article will be available on ScienceDirect shortly after the corrections to the proofs have been received by Elsevier. I suggest that you read them carefully and check them once more against the original; you are the only one responsible for doing this. Together with the proofs you will receive an order form for reprints.

Editorial policy is to publish all papers quickly but authors will appreciate that the desire to have issues with balanced contents implies that papers will not simply appear in the order of their acceptance. That and the need to publish at a uniform monthly rate when the supply is variable will occasionally delay a paper's appearance longer than the Editors would wish, but if this should be the case with your paper, we trust that we can count on your understanding.

Your paper from now on is no longer under my control. Please, address queries only to the Journal Manager:

Kate Partner

E-Mail: K.Partner@elsevier.com

When your paper is published on ScienceDirect, you want to make sure it gets the attention it deserves. To help you get your message across, Elsevier has developed a new, free service called AudioSlides: brief, webcast-style presentations that are shown (publicly available) next to your published article. This format gives you the opportunity to explain your research in your own words and attract interest. You will receive an invitation email to create an AudioSlides presentation shortly. For more information and examples, please visit http://www.elsevier.com/audioslides.

Yours sincerely,

Robert Graham Dyson, PhD

Editor

European Journal of Operational Research 